Ann. Biol. anim. Bioch. Biophys., 1979, 19 (3 B), 911-914.

\title{
Apport du traitement d'images radiologiques à l'étude du transit digestif : cinétique quantitative comparée d'une suspension barytée avec ou sans glucides chez le rat éveillé
}

\author{
par Odette MORELLE, C. GAUDEAU, J. RIVAILLIER *, J. THOUVENOT \\ Laboratoire de Physiologie, Faculté de Médecine \\ 2 bis, bd Tonnellé, 37000 Tours.
}

Summary. A study of digestive transit using X-ray picture analysis : Quantitative comparative kinetics of baryum sulfide with or without carbohydrates in conscious rats.

Pictures were taken at different stages of total digestive transit. These pictures were automatically analyzed for a study of volumetric and biochemical food factors. The mean gastric outflow generally depended on initial gastric volume and the energetic value of the meal. However, the transit time varied in the small intestine according to the nature of the carbohydrate.

Nous nous sommes proposés, en utilisant une méthode originale de traitement d'images (Morelle, Gaudeau et Thouvenot, 1978), de reprendre l'étude radiologique du transit chez le rat éveillé en posant le problème de la part respective des stimulus volumétriques (volume ingéré) et de l'ensemble des stimulus biochimiques alimentaires dans les régulations déterminant les conditions de l'absorption optimale. La présente étude ne concerne que le transit de suspensions barytées comportant ou non des glucides (glucose, lactose, saccharose).

\section{Technique.}

Les animaux, rats mâles Wistar 350 à $450 \mathrm{~g}$, sont mis à jeun $20 \mathrm{~h}$ environ avant l'expérience : on autorise l'apport hydrique ad libitum.

La suspension barytée est introduite dans l'estomac à l'aide d'une seringue munie d'un cathéter. On emploie une suspension d'Actybaryte (Derol), soit seule, soit addi-

* Travail effectué en collaboration avec le Laboratoire des Prototypes du C.N.R.S. Nous tenons à remercier $M$. Gazeau pour son assistance informatique. 
tionnée de glucose, de lacłose ou de saccharose, à raison de $0,5 \mathrm{~g}$ de poudre par $\mathrm{ml}$ de suspension. Les volumes administrés sont 2,3 et $4 \mathrm{ml}$.

L'animal est alors placé dans une enceinte adaptée à la radiographie et à laquelle il a été habitué. Les clichés correspondent aux temps suivants : $0,5,1,2,3,4,5,10$, $15,30,45,60 \mathrm{mn}$ puis d'heure en heure jusqu'à la fin du transit.

- Pour le traitement des images, on a utilisé un système d'analyse de photographies et d'images radiologiques mis au point au Service des Prototypes du C.N.R.S. La densitométrie permet d'intégrer l'opacité globale de la masse viscérale. On peut à l'aide de masques appropriés, étudier l'évolution de l'opacité correspondant à l'estomac, au grêle et au côlon (fig. 1). La chaîne permet de visualiser sur écran

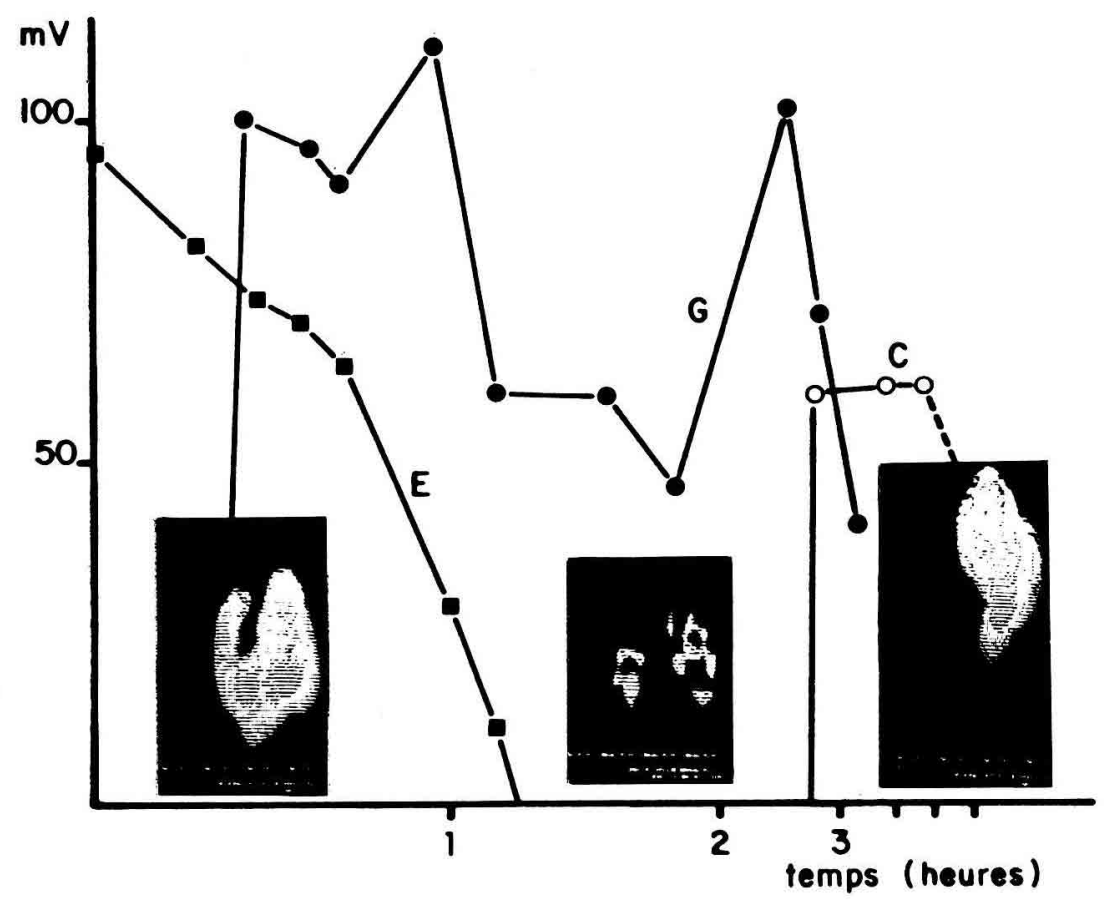

FIG. 1. - Diagramme montrant l'évolution au cours du transit baryfé de l'opacité globale de l'image de l'estomac E, de l'intestin grêle $\mathrm{G}$ ef du côlon C (Temps sur échelle log., ordonnées signal exprimé en $\mathrm{mV}$ ). Sur ce diagramme sont représentés, à titre indicatif, 3 exemples des images digitalisées restituées en niveaux de gris sur l'écran de visualisation.

l'image digitalisée et représentée au moyen de caractères choisis selon les différents niveaux de transparence. Pour chaque secteur, un programme automatique permet d'obtenir l'histogramme des niveaux et la surface correspondante. L'image gastrique est traitée séparément de celle du grêle. Pour ce dernier, étant donné la complexité des clichés, nous avons été conduits à diviser l'étendue maximale de l'image en 16 régions. L'histogramme des niveaux nous permet de connaître la répartition de la masse barytée dans chaque région et pour chaque temps. Afin d'essayer de reconstituer 
les transferts d'une région dans l'aułre, nous proposons une représentation qui s'inspire des machines séquentielles (Thouvenot, Gaudeau et Penaud, 1971) (fig. 2).

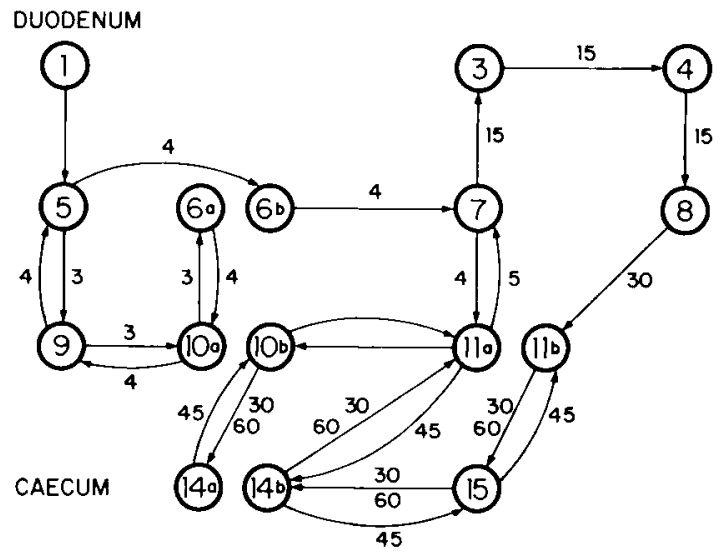

FIG. 2. - Représentation du graphe des états d'occupation par la baryfe des zones de l'étendue maximale de l'image selon un quadrillage orthogonal (chiffres entourés de 1 à 16 ; numérotation de gauche à droite et de haut en bas). Plusieurs zones comprennent deux sous-images traversées $a$ et $b$. Les chiffres à côté des flèches représentent les temps de transit en minutes.

Les données relatives au transit global sont (tabl. 1) :

- Présence de contrainte ou d'inhibition retardant le début de la vidange (côté 0 ou $+)$.

- Présence de variations d'homogénéité de l'image gastrique (oscillations du tonus cotées de 0 à 2 ).

- Les temps : $t_{0,5 G}$ (demi-temps) ef $t_{G t}$ (temps total) d'évacuation gastrique.

- Le début de réplétion du grêle $t_{0}$ J.

TABLEAU 1

\begin{tabular}{|c|c|c|c|c|c|c|c|c|c|c|c|}
\hline Substanc & & $\begin{array}{c}\text { Con- } \\
\text { trainte }\end{array}$ & $\begin{array}{l}\text { Oscil- } \\
\text { lations } \\
\text { du tonus }\end{array}$ & $t_{0,5} \mathrm{G}$ & $t_{C: t}$ & $t_{0} J$ & $J_{+}$ & $\mathrm{J}_{\mathrm{mux}}$ & $t_{J I}$ & $t_{\mathrm{CO}}$ & $t_{C R}$ \\
\hline Baryte & $\begin{array}{l}2 \mathrm{ml} \\
3 \mathrm{ml} \\
4 \mathrm{ml}\end{array}$ & $\begin{array}{l}0 \\
0 \\
0\end{array}$ & $\begin{array}{l}1 \\
1 \\
1\end{array}$ & $\begin{array}{r}10 \mathrm{mn} \\
10 \mathrm{mn} \\
3 \mathrm{a} 4 \mathrm{mn}\end{array}$ & $\begin{array}{c}30-45 \mathrm{mn} \\
30-45 \mathrm{mn} \\
15 \mathrm{mn}\end{array}$ & $\begin{array}{l}2 \mathrm{mn} \\
2 \mathrm{mn} \\
2 \mathrm{mn}\end{array}$ & $\begin{array}{r}10 \mathrm{mn} \\
10 \mathrm{mn} \\
5 \mathrm{mn}\end{array}$ & $\begin{array}{l}30-45 \\
45 \mathrm{mn} \\
10 \mathrm{mn}\end{array}$ & $\begin{array}{l}3 \mathrm{~h} \\
3 \mathrm{~h} \\
4 \mathrm{~h}\end{array}$ & $\begin{array}{l}2 \mathrm{~h} \\
2 \mathrm{~h} \\
2 \mathrm{~h}\end{array}$ & $\begin{array}{l}5 h 30 \\
5 h \\
6 h\end{array}$ \\
\hline $\begin{array}{l}\text { Glucose } \\
1,67 \mathrm{KJ}\end{array}$ & $\begin{array}{c}2 \mathrm{ml} \\
\text { ou } \\
4 \mathrm{ml}\end{array}$ & $\begin{array}{l}t \\
0\end{array}$ & $\begin{array}{l}2 \\
0\end{array}$ & $\begin{array}{l}10 \mathrm{mn} \\
10 \mathrm{mn}\end{array}$ & $\begin{array}{l}1 \mathrm{~h} \\
45 \mathrm{mn}\end{array}$ & $\begin{array}{l}2 \mathrm{mn} \\
5 \mathrm{mn}\end{array}$ & $\begin{array}{l}2-4 \mathrm{mn} \\
10 \mathrm{mn}\end{array}$ & $\begin{array}{l}15 \mathrm{mn} \\
30 \mathrm{mn}\end{array}$ & $\begin{array}{l}2 \mathrm{~h} \\
2 \mathrm{~h}\end{array}$ & $\begin{array}{l}2 \mathrm{~h} \\
2 \mathrm{~h}\end{array}$ & $\begin{array}{l}6 h \\
6 h\end{array}$ \\
\hline $\begin{array}{l}\text { Lactose } \\
3,34 \mathrm{KJ} \\
\text { Saccharose } \\
3,34 \mathrm{KJ} \\
\text { Saccharose } \\
1,67 \mathrm{KJ}\end{array}$ & $\begin{array}{l}2 \mathrm{ml} \\
2 \mathrm{ml} \\
2 \mathrm{ml}\end{array}$ & $\begin{array}{l}-1 \\
0\end{array}$ & $\begin{array}{c}-t-t- \\
t-t- \\
0\end{array}$ & $\begin{array}{c}30 \mathrm{mn} \\
15-30 \mathrm{mn} \\
30 \mathrm{mn}\end{array}$ & $\begin{array}{c}3 h \\
3-4 h \\
1 \mathrm{~h} 30 \mathrm{mn}\end{array}$ & $\begin{array}{l}5 \mathrm{mn} \\
2 \mathrm{mn} \\
2 \mathrm{mn}\end{array}$ & $\begin{array}{l}10 \mathrm{mn} \\
30 \mathrm{mn} \\
30 \mathrm{mn}\end{array}$ & $\begin{array}{c}30 \mathrm{mn} \\
1 \mathrm{~h} \\
1 \mathrm{~h}\end{array}$ & $\begin{array}{l}3 \mathrm{~h} \\
5 \mathrm{~h} \\
4 \mathrm{~h}\end{array}$ & $\begin{array}{l}3 \mathrm{~h} \\
3 \mathrm{~h} 30 \\
3 \text { h } 30\end{array}$ & $\begin{array}{l}6 \text { h } 30 \\
5 \text { h } 30 \\
5 \text { h } 30\end{array}$ \\
\hline
\end{tabular}


- Le temps correspondant à l'apparition d'anses grêles sur une étendue de 50 p. 100 $\left(J_{+}\right)$et 100 p. $100\left(J_{\max }\right)$.

- Le temps total de réplétion du grêle $\left(t_{J I}\right)$, le temps cæcal $\left(t_{\mathrm{CO}}\right)$ et le temps marquant le début d'apparition des crottes $\left(\mathfrak{t}_{\mathrm{CR}}\right)$.

\section{Résultats.}

En général le temps global de transit varie peu ( 5 h 30 à 6 h 30) quel que soit le type de substance. En revanche des variations importantes portent sur le temps de vidange gastrique : pour la baryte seule le volume décroît linéairement en fonction du temps; le volume initial influe sur la pente de vitesse $-K\left(-K\right.$ proportionnel à $\left.V_{0}\right)$. Pour les mélanges de substances digestibles, la cinétique est du type exponentiel décroissant : la valeur énergétique paraît influer notablement sur le temps.

Toutefois, la comparaison glucose-saccharose à doses isoénergétiques montre quavec ce dernier on observe encore un léger ralentissement du transit.

\section{Discussion.}

L'examen des résultats suggère que le transport de substances inertes ne paraît dépendre que des grandeurs biomécaniques : gradient de pression, volume initial, résistances d'écoulement. Des mécanismes régulateurs de la motricité peuvent s'organiser à partir des mécano-récepteurs (volorécepteurs notamment, Mei, 1970).

Pour les aliments digestibles interviennent, outre le volume initial intra-gastrique, la valeur énergétique (Hunt et Stubbs, 1975). Enfin, le traitement d'image a pu mettre en évidence pour le saccharose une phase de stagnation iléale qui pourrait être en relation avec des sites d'absorption.

Commission CNERNA Digestion-Absorption/Association des Physiologistes, Paris 5-6 octobre 1978.

\section{Références}

HUNT J. N., MACDONALD I., 1954. The influence of volume on gastric emptying. J. Physiol., 126, 459-474.

HUNT J. N., STUBBS D. F., 1975. The volume and energy content of meals as determinant of gastric emplying. J. Physiol., 245, 209-225.

MEI N., 1970. Mécanorécepteurs vagaux digestifs chez le chat. Exp. Brain Res., 11, 502-514.

MORELLE O., GAUDEAU C., THOUVENOT J., 1978. Apport du traitement d'images radiologiques à l'étude du transit digestif. Biosigma, 2, 238-244.

THOUVENOT J., GAUDEAU C., PENAUD J., 1971. Reconnaissances des états d'un système physiologique par des automates séquentiels. Application à l'analyse des processus moteurs digestifs. Symp. d'Informatique médicale, Saint-Lary, Editions I. R. I. A. 